\title{
24-h ambulatory recording of aortic pulse wave velocity and central systolic augmentation: a feasibility study
}

\author{
Leonella Luzardo ${ }^{1,2}$, Inés Lujambio ${ }^{1,2}$, Mariana Sottolano ${ }^{1,2}$, Alicia da Rosa ${ }^{1,2}$, Lutgarde Thijs ${ }^{3}$, Oscar Noboa ${ }^{1}$, \\ Jan A Staessen ${ }^{3,4}$ and José Boggia ${ }^{1,2}$
}

We assessed the feasibility of ambulatory pulse wave analysis by comparing this approach with an established tonometric technique. We investigated 35 volunteers (45.6 years; $51.0 \%$ women) exclusively at rest ( $R$ study) and 83 volunteers (49.9 years; $61.4 \%$ women) at rest and during daytime $(1000-2000 \mathrm{~h})$ ambulatory monitoring $(\mathrm{R}+\mathrm{A}$ study). We recorded central systolic (cSP), diastolic (cDP) and pulse (cPP) pressures, augmentation index (cAl) and pulse wave velocity (PWV) by brachial oscillometry (Mobil-0-Graph 24h PWA Monitor) and radial tonometry (SphygmoCor). We applied the Bland and Altman's statistics. In the R study, tonometric and oscillometric estimates of cSP (105.6 vs. $106.9 \mathrm{~mm} \mathrm{Hg}), \mathrm{cDP}(74.6 \mathrm{vs} .74 .7 \mathrm{~mm} \mathrm{Hg})$, cPP $(31.0$ vs. $32.1 \mathrm{~mm} \mathrm{Hg})$, cAl $(21.1$ vs. $20.6 \%)$ and PWV $\left(7.3\right.$ vs. $\left.7.0 \mathrm{~m} \mathrm{~s}^{-1}\right)$ were similar $(P \geqslant 0.11)$. In the $\mathrm{R}+\mathrm{A}$ study, tonometric vs. oscillometric assessment yielded similar values for cSP $(115.4$ vs. $113.9 \mathrm{~mm} \mathrm{Hg} ; P=0.19)$ and cAl (26.5 vs. $25.3 \% ; P=0.54)$, but lower cDP $(77.8$ vs. $81.9 \mathrm{~mm} \mathrm{Hg} ; \boldsymbol{P}<0.0001)$, so that cPP was higher $(37.6$ vs. $32.1 \mathrm{~mm} \mathrm{Hg}$; $P<0.0001)$. PWV (7.9 vs. $\left.7.4 \mathrm{~m} \mathrm{~s}^{-1}\right)$ was higher $(P=0.0002)$ on tonometric assessment. The differences between tonometric and oscillometric estimates increased $(P \leqslant 0.004)$ with $\operatorname{CSP}(r=0.37)$, cAl $(r=0.39)$ and PWV $(r=0.39)$, but not $(P \geqslant 0.17)$ with $\mathrm{CDP}(r=0.15)$ or $\mathrm{CPP}(r=0.13)$. Irrespective of measurement conditions, brachial oscillometry compared with an established tonometric method provided similar estimates for CSP and systolic augmentation, but slightly underestimated PWV. Pending further validation, ambulatory assessment of central hemodynamic variables is feasible.

Hypertension Research (2012) 35, 980-987; doi:10.1038/hr.2012.78; published online 24 May 2012

Keywords: ambulatory monitoring; arterial stiffness; central augmentation; central blood pressure; pulse wave velocity

\section{INTRODUCTION}

Arterial stiffness predicts cardiovascular complications over and beyond traditional risk factors not only in patients with hypertension, diabetes mellitus or previous cardiovascular disease, but in subjects randomly recruited from populations as well. ${ }^{1}$ Experts consider aortic pulse wave velocity (PWV) as the gold standard in the assessment of arterial stiffness. ${ }^{2}$ Pulse wave analysis is an alternative approach to quantify arterial stiffness. The arterial pressure wave consists of a forward component generated by the heart and reflected waves returning to the heart from peripheral sites. As the arteries become stiffer with advancing age, the reflected waves return faster, reach the proximal aorta during systole and cause augmentation of late systolic blood pressure (SBP). ÓRourke and colleagues ${ }^{3}$ developed a simple and reproducible tonometric method to assess various indices of arterial stiffness. A validated algorithm permits transformation of peripheral arterial to central aortic waveforms. ${ }^{3-5}$ Analysis of the shape and timing of these waveforms provides information on central pulse pressure (cPP) and augmentation.
The Mobil-O-Graph 24h PWA Monitor (I.E.M. GmbH, Stolberg, Germany) is a validated monitor for 24 -h blood pressure monitoring. ${ }^{6,7}$ It includes the ARCSolver application, ${ }^{8}$ which allows pulse wave analysis of the central blood pressure and measuring of aortic PWV. We conducted a study in Uruguayan volunteers to compare central hemodynamic measurements obtained by the Mobil-O-Graph at rest and under ambulatory conditions with those obtained by the SphygmoCor (AtCor Medical, West Ryde, New South Wales, Australia) at rest.

\section{METHODS}

Recruitment of volunteers

We recruited volunteers among staff and outpatients at the Manuel Quintela University Hospital, Montevideo, Uruguay, from 1 November 2010 to 11 March 2011. We announced the study by e-mails, flyers, posters and word of mouth. To be eligible, volunteers had to be in sinus rhythm without extrasystoles, able bodied without debilitating cardiovascular or non-cardiovascular disease and had to provide informed written consent. Stage-1 hypertension,

${ }^{1}$ Unidad de Hipertensión Arterial and Centro de Nefrología, Hospital de Clínicas, Universidad de la República, Montevideo, Uruguay; ${ }^{2}$ Departamento de Fisiopatología, Hospital de Clínicas, Universidad de la República, Montevideo, Uruguay; ${ }^{3}$ Studies Coordinating Centre, Department of Cardiovascular Diseases, Division of Hypertension and Cardiovascular Rehabilitation, University of Leuven, Leuven, Belgium and ${ }^{4}$ Department of Epidemiology, Maastricht University, Maastricht, The Netherlands Correspondence: Dr J Boggia, Unidad de Hipertensión Arterial, Hospital de Clínicas Dr Manuel Quintela, Av Italia 2870 Piso 15 Sala 1 Ap 13, PO-11600 Montevideo, Uruguay, E-mail: jboggia@hc.edu.uy

Received 25 August 2011; revised 26 January 2012; accepted 2 April 2012; published online 24 May 2012 
stable type-2 diabetes mellitus, treatment with antihypertensive drugs or oral antidiabetic agents and obesity did not lead to exclusion. The Ethics Committee of the University Hospital approved the study.

\section{Clinical measurements}

To ensure a steady-state condition, we did the hemodynamic measurements in a quiet examination room at the Hypertension Unit. Subjects were asked to refrain from smoking, heavy exercise, drinking alcohol, caffeine-containing beverages or maté (llex paraguariensis) for at least $2 \mathrm{~h}$ before the examination. Peripheral blood pressure was the average of three consecutive readings, obtained at the upper arm after the volunteers had rested for at least $5 \mathrm{~min}$ in the sitting position using a validated OMRON 705IT oscillometric sphygmomanometer (Omron Healthcare, Kyoto, Japan). For all blood pressure measurements, we used large cuffs if arm circumference was $\geqslant 31 \mathrm{~cm}$ and standard cuffs otherwise. Hypertension was a brachial blood pressure of at least $140 \mathrm{~mm} \mathrm{Hg}$ systolic or $90 \mathrm{~mm} \mathrm{Hg}$ diastolic or the use of antihypertensive drugs. We measured body weight by means of an OMRON HBF-500INT scale with the subjects wearing light indoor clothing without shoes. Height was measured to the nearest $0.5 \mathrm{~cm}$ with the subjects stretching against a wall. Body mass index was weight in kilogram divided by the square of height in meters. We administered a standardized questionnaire to obtain information on each subject's medical history, smoking and drinking habits and use of medications.

\section{Tonometric measurements at the radial artery}

After the participants had rested $15 \mathrm{~min}$ in the supine position, we recorded during an 8-s period the radial waveform at the right radial artery. We used a high-fidelity SPC-301 micromanometer (Millar Instruments, Houston, TX) interfaced with a computer running SphygmoCor software, version 8.2 (AtCor Medical, West Ryde, New South Wales, Australia). We discarded recordings when the systolic or diastolic variability of consecutive waveforms exceeded $5 \%$, the pulse height variation exceeded $5 \%$ or the amplitude of the pulse wave signal was $<80 \mathrm{mV}$. We calibrated the pulse wave by measuring the brachial blood pressure immediately before the SphygmoCor readings.

From the radial signal, the SphygmoCor software calculates the aortic pulse wave by means of a validated and population-based generalized transfer function. ${ }^{3-5}$ The peripheral (radial) augmentation index (AI) was defined as the ratio of the second to the first peak of the pressure wave expressed in percent. The central (aortic) AI was the difference between the second and first systolic peaks given as a percentage of the aortic pulse pressure. Peripheral and central pulse pressures were the differences of SBP minus diastolic blood pressure (DBP) derived from the brachial blood pressure and from the aortic pulse wave, respectively. Aortic PWV was measured by sequential ECG-gated recordings of the arterial pressure waveform at the carotid and femoral arteries. Distances from the suprasternal notch to the carotid sampling site (distance A) and from the suprasternal notch to the femoral sampling site (distance B) were measured. Pulse wave travel distance was calculated as distance $B$ minus distance A. Pulse transit time was the average of 10 consecutive beats. PWV was the distance in meters divided by the transit time in seconds.

\section{Oscillometric measurements at the brachial artery}

We programmed oscillometric Mobil-O-Graph 24h PWA Monitor monitors (I.E.M. GmbH, Stolberg, Germany), ${ }^{6,7}$ fitted with the same cuff size as for the office blood pressure measurements, to obtain readings with an interval of $20 \mathrm{~min}$ from $0700 \mathrm{~h}$ until $2300 \mathrm{~h}$ and every $30 \mathrm{~min}$ from $2300 \mathrm{~h}$ until $0700 \mathrm{~h}$. If the ambulatory recordings were longer than 1 day, only the first $24 \mathrm{~h}$ were analyzed. Intra-individual means of the ambulatory measurements were weighted by the time interval between successive readings. ${ }^{9}$ The runs test with an one-sided probability of $5 \%$ was used to differentiate a diurnal rhythm from random variability in the hemodynamic measurements within individuals..$^{10}$ All the recordings took place during working days.

The ARCSOlver algorithm, as implemented in Mobil-O-Graph 24h PWA Monitor, reconstructs the central pulse wave by applying a transfer function. ${ }^{8}$ The recordings are carried out at the diastolic pressure level for approximately $10 \mathrm{~s}$, using a conventional blood pressure cuff for adults available in two sizes $(24-34 \mathrm{~cm}$ and $32-42 \mathrm{~cm})$ and a high-fidelity pressure sensor (MPX5050, Freescale, Tempe, AZ, USA). The transfer function implemented in the
ARCSolver software of the Mobil-O-Graph 24h PWA Monitor includes an algorithm for checking quality of the signal. The quality is graded from 1 to 4 . Results of excellent or good quality are labeled 1 and 2 and include, respectively, $>80$ or $50 \%$ of the cardiac cycles during signal acquisition. Grade 3 results are estimated from $<50 \%$ of the recorded cycles and are considered to be of poor quality. Grade 4 indicates missing results because of insufficient signal quality. We analyzed central hemodynamic measurements only if graded 1 or 2 . The central aortic pressure waveform is decomposed into forward and reflected waves using an uncalibrated triangular aortic flow waveform. PWV is estimated from the time difference between the derived forward and reflected waves after signal processing based on waveform constraint criteria. The model used is linear with a continuous parameter space for arterial resistance, peripheral resistance and arterial compliance. The ARCSolver software computes aortic PWV from the reconstructed central pulse wave, characteristic impedance ${ }^{11}$ and age, ${ }^{8}$ based on the concept that the ejection work of the left ventricle is subject to an optimization principle, ${ }^{8,12}$ and assuming a 3-element Windkessel model. ${ }^{11}$

\section{Studies exclusively at rest in the laboratory $-\mathrm{R}$ study}

In studies at rest recumbent, we compared in an air-conditioned laboratory the new method (Mobil-O-Graph) with an established technique (SphygmoCor) to assess central blood pressure and aortic PWV, while minimizing the influence of body position, physical activity, temperature, and the type of monitor to calibrate the central wave form (Figure 1). We examined 35 volunteers after they had rested for $15 \mathrm{~min}$ in the supine position. We obtained measurements of the brachial blood pressure and the central hemodynamic variables at baseline and after 15, 30 and $45 \mathrm{~min}$ by means of the Mobil-OGraph with an appropriately sized cuff applied to the left arm of the participants. The four readings so obtained were averaged for analysis. We also measured the central hemodynamic variables by means of the SphygmoCor device between the first and second and between the third and fourth Mobil-O-Graph reading. We calibrated the SphygmoCor using the brachial blood pressure as reported by the Mobil-O-Graph at baseline and at $30 \mathrm{~min}$. To exclude interference of the cuff inflation with the SphygmoCor measurements, we recorded the tonometric signal over the radial artery at the recommended right arm. ${ }^{13}$ For analysis, we averaged the two SphygmoCor measurements.

\section{Studies at rest in the office and under ambulatory conditions- Study $\mathbf{R}+\mathbf{A}$}

We enrolled 83 volunteers in a second study, in which we compared the central hemodynamic variables as measured by the Mobil-O-Graph under 'real life'

a

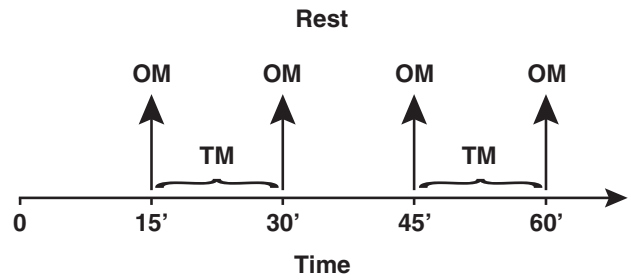

b

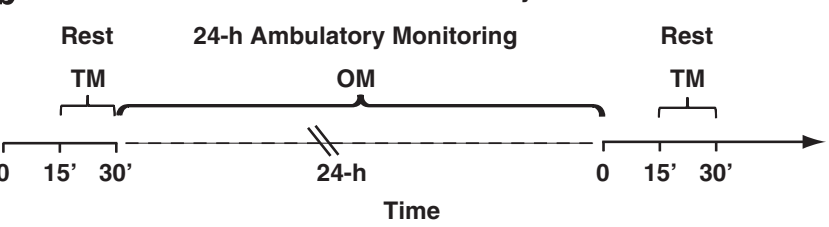

Figure 1 Description of the two protocols. (a) $\mathrm{R}$ refers to studies exclusively in the office at rest of 35 participants. (b) $R+A$ refers to the studies at the office at rest and in ambulatory conditions of 83 participants. Tonometry (TM) was done at the radial artery by means of the SphygmoCor and oscillometry (OM) at the brachial by means of the Mobil-O-Graph. The SphygmoCor was calibrated by the brachial blood pressure obtained by the Mobil-O-Graph in the study R and by the OMRON 705/T in the study R+A. 
ambulatory conditions with those obtained by the SphygmoCor at rest in the laboratory (Figure 1). The SphygmoCor measurements were done at the right radial artery after the participants had rested $15 \mathrm{~min}$ in the recumbent position and were calibrated against the brachial blood pressure as measured by the OMROM 705IT at the same arm. ${ }^{13}$ After the SphygmoCor measurements, the Mobil-O-Graph recordings were initiated and ran over at least $24 \mathrm{~h}$. When the participants came back the next day to return the Mobil-O-Graph monitors, the SphygmoCor measurements were repeated. For comparison of the techniques, we averaged the SphygmoCor measurements obtained at the initiation and termination of the ambulatory recordings and we used the daytime ambulatory measurements. Daytime spanned the interval from 1000 to $2000 \mathrm{~h}$ and therefore excluded the morning and evening periods of the diurnal profile, during which blood pressure changes rapidly in most subjects.

\section{Statistical methods}

For database management and statistical analysis, we used SAS software, version 9.1.3 (SAS Institute, Cary, NC). For comparison of means and proportions, we applied the Student's $t$-test for paired observations and the $\chi^{2}$ statistic, respectively. We assessed the agreement between paired measurements by the Bland and Altman's method. ${ }^{14}$ Reproducibility was twice the standard deviation of the pairwise differences between duplicate measurements, expressed as a percentage of the average of first and repeat measurements in all participants. ${ }^{14}$ Using single regression, we also assessed the departure of the slope of repeated on first measurements from the line of identity $($ slope $=1)$.

\section{RESULTS}

\section{Characteristics of participants}

The 35 participants enrolled in the $\mathrm{R}$ study included 18 women (51.0\%; Table 1). Their age averaged ( \pm s.d.) $45.6 \pm 16.4$ years (range 23-78 years). SBP and DBP in the 35 volunteers, as measured at the office, averaged $119.8 \pm 18.1$ and $70.2 \pm 12.0 \mathrm{~mm} \mathrm{Hg}$, and heart rate $65.0 \pm 9.8$ beats per minute.

Of the 83 volunteers participating in the $\mathrm{R}+\mathrm{A}$ study, 51 (61.4\%) were female (Table 1 ). Their age averaged ( \pm s.d.) $49.9 \pm 13.1$ years (range 20-80 years). Blood pressure on office measurement averaged $126.5 \pm 15.6 \mathrm{~mm} \mathrm{Hg}$ systolic and $76.8 \pm 10.6 \mathrm{~mm} \mathrm{Hg}$ diastolic and $119.3 \pm 12.1$ and $77.2 \pm 9.0 \mathrm{~mm} \mathrm{Hg}$ on 24 -h ambulatory monitoring. The corresponding heart rates were $68.8 \pm 10.4$ and $75.1 \pm 8.7$ beats per minute, respectively. Of the 83 volunteers, 46 (55.4\%) were hypertensive, $5(6.0 \%)$ had diabetes, $17(20.5 \%)$ were current smokers, and $45(54.2 \%)$ reported drinking alcohol once a week or more frequently. Of the hypertensive patients, $34(73.9 \%)$ were on antihypertensive drug treatment with diuretics $(n=10), \beta$-blockers $(n=6)$, calcium channel blockers $(n=6)$, inhibitors of the renin system $(n=28)$ or other drugs $(n=1)$. Table 1 provides the characteristics of the participants by study and sex. Women enrolled in the R + A study, compared with men, were slightly older, had lower SBP and DBP on office and 24-h ambulatory measurement and less frequently reported regular alcohol intake.

The median number of blood pressure readings per participant during 24-h ambulatory monitoring was 50 (interquartile range 41-56). The median number of good-quality central hemodynamic readings per participant was 37 (interquartile range 26-43). Figure 2 shows the diurnal profiles of the central hemodynamic measurements in the 83 participants enrolled in the $\mathrm{R}+\mathrm{A}$ study. We observed a significant difference between daytime and nighttime values in 63 participants (75.9\%) for SBP and DBP, in 20 participants $(24.1 \%)$ for pulse pressure, in 32 participants $(38.5 \%)$ for the crude $\mathrm{AI}$, in 41 participants $(49.4 \%)$ for the AI standardized to 75 beats per minute and in 47 participants $(56.6 \%)$ for PWV.
Table 1 Baseline characteristics of participants by study and sex

\begin{tabular}{lcccccc}
\hline \multirow{2}{*}{ Characteristics } & Women & Men & & Studies exclusively in the & & \multicolumn{2}{c}{$\begin{array}{c}\text { Studies at the office and in } \\
\text { laboratory at rest }(R)\end{array}$} & & Wmbulatory conditions $(R+A)$ & Men \\
\cline { 2 - 3 } Number & 18 & 17 & & 51 & 32
\end{tabular}

\section{Anthropometrics}

$\begin{array}{lcccc}\text { Age, years } & 45.7 \pm 16.1 & 45.5 \pm 17.3 & 52.2 \pm 12.3 & 45.8 \pm 13.6^{*} \\ \text { Height, cm } & 157.2 \pm 5.2 & 171.4 \pm 9.1^{\ddagger} & 157.0 \pm 5.9 & 171.8 \pm 6.1^{\ddagger} \\ \text { Weight, kg } & 61.0 \pm 8.0 & 74.7 \pm 11.6^{\ddagger} & 70.4 \pm 13.8 & 88.4 \pm 17.9^{\ddagger} \\ \text { Body mass index, } & 24.8 \pm 3.9 & 25.4 \pm 3.3 & 28.5 \pm 5.0 & 30.0 \pm 5.6\end{array}$

$\mathrm{kg} \mathrm{m}^{-2}$

\begin{tabular}{|c|c|c|c|c|}
\hline \multicolumn{5}{|l|}{ Risk factors } \\
\hline $\begin{array}{l}\text { Current smoking, } \\
n(\%)\end{array}$ & $4(22.2)$ & $6(35.3)$ & $12(23.5)$ & $5(15.6)$ \\
\hline $\begin{array}{l}\text { Alcohol intake, } \\
n(\%)\end{array}$ & $5(27.8)$ & $9(52.9)$ & $21(41.2)$ & $24(75.0)^{\dagger}$ \\
\hline $\begin{array}{l}\text { Hypertension, } \\
n(\%)\end{array}$ & $5(27.8)$ & $1(6.0)$ & $32(62.7)$ & $14(43.7)$ \\
\hline $\begin{array}{l}\text { Treated hyperten } \\
\text { sion, } n(\%)\end{array}$ & $0(0)$ & $1(6.0)$ & $24(47.1)$ & $10(31.2)$ \\
\hline Diabetes, $n(\%)$ & $2(11.1)$ & $1(6.0)$ & $3(5.8)$ & $2(6.2)$ \\
\hline \multicolumn{5}{|l|}{ Office measurement } \\
\hline $\begin{array}{l}\text { Systolic pressure, } \\
\mathrm{mm} \mathrm{Hg}\end{array}$ & $114.5 \pm 20.2$ & $125.4 \pm 14.0$ & $122.4 \pm 15.8$ & $132.9 \pm 13.1^{\dagger}$ \\
\hline $\begin{array}{l}\text { Diastolic pres- } \\
\text { sure, } \mathrm{mm} \mathrm{Hg}\end{array}$ & $68.3 \pm 11.2$ & $72.1 \pm 12.8$ & $74.7 \pm 10.1$ & $80.4 \pm 10.1^{*}$ \\
\hline $\begin{array}{l}\text { Pulse pressure, } \\
\mathrm{mm} \mathrm{Hg}\end{array}$ & $46.2 \pm 10.3$ & $53.2 \pm 8.4^{*}$ & $47.7 \pm 9.6$ & $51.7 \pm 9.6^{*}$ \\
\hline Heart rate, bpm & $66.87 \pm 10.4$ & $62.78 \pm 8.54^{*}$ & $68.6 \pm 10.2$ & $69.0 \pm 10.8$ \\
\hline
\end{tabular}

\section{4-Hambulatory recordings}

Systolic pressure, $\quad$...

$\mathrm{mm} \mathrm{Hg}$

Diastolic pres-

sure, $\mathrm{mm} \mathrm{Hg}$

Pulse pressure,

$\mathrm{mm} \mathrm{Hg}$

Heart rate, bpm

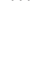

$\cdots$

$116.7 \pm 12.4 \quad 123.4 \pm 10.3^{\dagger}$

$74.7 \pm 8.9 \quad 81.3 \pm 7.7^{\dagger}$

$42.0 \pm 7.8 \quad 42.1 \pm 5.7$

$74.7 \pm 8.4 \quad 75.6 \pm 9.4$

Values are mean \pm s.d. or number of subjects (\%). An ellipsis indicates information that was not collected. Hypertension was an office blood pressure of at least $140 \mathrm{~mm} \mathrm{Hg}$ systolic or $90 \mathrm{~mm} \mathrm{Hg}$ diastolic or use of antihypertensive drugs. Significance of the sex difference ${ }^{*} P<0.05,{ }^{\dagger} P<0.01$ and ${ }^{\ddagger} P<0.001$.

\section{Studies exclusively at rest in the laboratory- $\mathrm{R}$ Study}

In the 35 volunteers, the tonometrically measured central blood pressure averaged 105.6 $\pm 17.1 \mathrm{~mm} \mathrm{Hg}$ systolic and $74.6 \pm 10.8 \mathrm{~mm} \mathrm{Hg}$ diastolic. The AI, crude and standardised to a heart rate of 75 beats per minute, was $21.1 \pm 13.0 \%$ and $15.1 \pm 13.4 \%$. PWV was $7.3 \pm 1.9 \mathrm{~m} \mathrm{~s}^{-1}$. The corresponding values for the central hemodynamic variables as derived by oscillometry at rest by the Mobil-OGraph were $106.9 \pm 16.6 \mathrm{~mm} \mathrm{Hg}, 74.7 \pm 11.3 \mathrm{~mm} \mathrm{Hg}, 20.6 \pm 11.1 \%$, $14.0 \pm 12.5 \%$ and $7.0 \pm 2.2 \mathrm{~m} \mathrm{~s}^{-1}$. Table 2 lists the central hemodynamic variables by study, sex and technique of measurement.

The same observer did the first and repeat SphygmoCor recordings in the 35 participants. The intra-observer intra-session reproducibility ranged from 8.2 to $7.0 \%$ for the central blood pressures and were $59 \%$ 

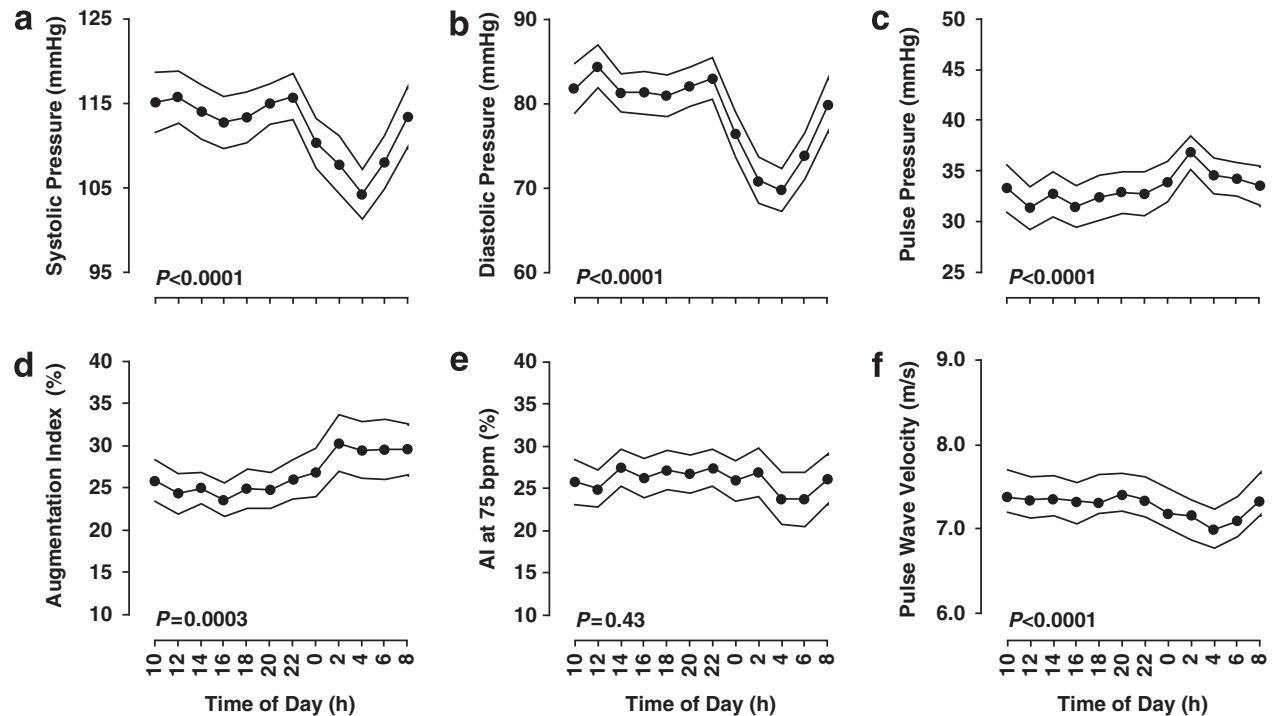

Figure 2 (a) Central systolic, (b) diastolic and (c) pulse pressures, the (d) crude and (e) standardised augmentation indices and for (f) aortic pulse wave velocity of 83 volunteers enrolled in the study $\mathrm{R}+\mathrm{A}$. Plotted values are 2 hourly mean with $95 \%$ confidence interval. $P$-values are for the comparison of the daytime (1000-2000 h) and nighttime (0000-0600 h) means.

Table 2 Central hemodynamic variables by study, sex and technique of measurement

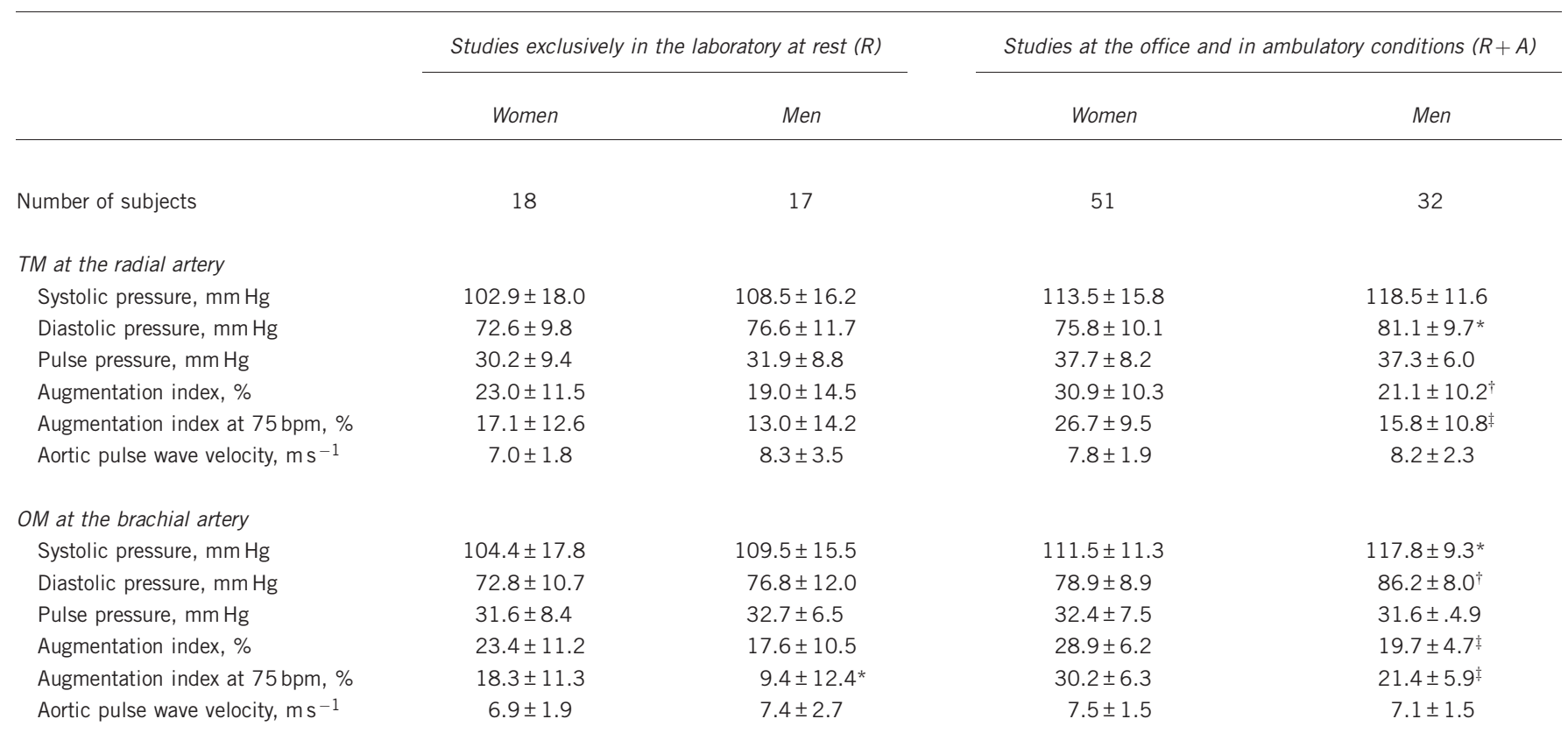

TM and OM refer to measurements obtained by the tonometric SphygmoCor technique at the radial artery or by the oscillometric Mobil-0-Graph monitor (1000-2000 hours) at the brachial artery, respectively. Values are mean \pm s.d. The number of measurements averaged per subjects were 2 for TM and 4 for OM in the R study and 2 for TM and $\geqslant 10$ for OM in the R+A study (Figure 1 ). Significance of the sex difference: ${ }^{*} P<0.05,{ }^{\dagger} P<0.01$ and ${ }^{\ddagger} P<0.001$.

and $16.4 \%$ for the crude $\mathrm{AI}$ and PWV, respectively. Expressed as a percentage of maximal variation (four times the standard deviation of the average of the repeat measurements), these values ranged $23.8-21.1 \%$ for the central blood pressures and were 29.4 and $17.1 \%$ for the $\mathrm{AI}$ and PWV, respectively. Expressing the reproducibility as a percentage of maximal variation allows comparing the reproducibility across measurements, higher values indicates lower concordance.
The Bland and Altman analysis of the R study appears in Table 3. The tonometric and oscillometric estimates of cSP (105.6 vs. $106.9 \mathrm{~mm} \mathrm{Hg})$, diastolic pressure $(74.6$ vs. $74.7 \mathrm{~mm} \mathrm{Hg})$ and pulse pressure $(31.0$ vs. $32.1 \mathrm{~mm} \mathrm{Hg})$ were similar $(P \geqslant 0.12)$. The crude AI (21.1 vs. $20.6 \% ; P=0.65$ ), the AI standardised to a heart rate of 75 beats per minute (15.1 vs. $14.0 \% ; P=0.57)$ and $\mathrm{PWV}$ (7.3 vs. 7.0 ; $P=0.11)$ were also similar. The Bland and Altman plots are given in Supplementary Figure $S 1$ available online. The $P$-values for 
between-method differences in women compared with men were all nonsignificant $(P \geqslant 0.14)$.

Studies at rest in the office and under ambulatory conditionsStudy $\mathbf{R}+\mathbf{A}$

The daytime (1000-2000 hours) ambulatory recordings included 2132 single blood pressure readings with good-quality estimates of the central hemodynamic variables in 1293 (60.6\%). Of the latter 1293 readings, $569(44.0 \%)$ were labeled by the Mobil-O-Graph as 'very good' and 724 (56.0\%) as 'good'. All volunteers completed a full 24-h ambulatory recording and did not report major discomfort owing to the second 10-s inflation of the cuff to diastolic pressure, which is required to measure the central hemodynamic variables.

In the 83 volunteers, the tonometrically measured central blood pressure averaged $115.4 \pm 14.5 \mathrm{~mm} \mathrm{Hg}$ systolic and $77.8 \pm 10.2 \mathrm{~mm} \mathrm{Hg}$ diastolic. The augmentation indices of crude and standardised to a heart rate of 75 beats per minute were $26.5 \pm 12.6$ and $22.2 \pm 11.5 \%$, respectively. PWV was $7.9 \pm 2.1 \mathrm{~m} \mathrm{~s}^{-1}$. The corresponding values for the central hemodynamic variables as derived by oscillometry during daytime oscillometric monitoring were $113.9 \pm 10.9 \mathrm{~mm} \mathrm{Hg}$, $81.9 \pm 9.2 \mathrm{~mm} \mathrm{Hg}, 25.3 \pm 7.2 \%, 26.8 \pm 7.5 \%$ and $7.4 \pm 1.6 \mathrm{~cm} \mathrm{~s}^{-1}$.

The same observer did the first and repeat SphygmoCor recordings in 79 participants. The intra-observer inter-session reproducibility ranged from 14.1 to $11.7 \%$ for the central blood pressures and were 60.7 and $24.2 \%$ for the AI and PWV, respectively. Expressed as a percentage of maximal variation (four times the standard deviation of the average of the repeat measurements), these values ranged $28.1-22.6 \%$ for the central blood pressures and were 29.0 and $22.3 \%$ for the AI and PWV, respectively.

The Bland and Altman analysis of the $\mathrm{R}+\mathrm{A}$ study appears in Table 4. Tonometric compared with oscillometric estimates of central hemodynamic variables provided similar estimates for cSP (115.4 vs. $113.9 \mathrm{~mm} \mathrm{Hg} ; P=0.19$ ), but $\mathrm{cDP}$ was lower on tonometric assessment $(77.8$ vs. $81.9 \mathrm{~mm} \mathrm{Hg} ; P<0.0001)$, so that $\mathrm{cPP}$ was higher $(37.6$ vs. $32.1 \mathrm{~mm} \mathrm{Hg} ; P<0.0001)$. Furthermore, the crude AI (26.5 vs. $25.3 \% ; P=0.54)$ was similar irrespective of the measurement technique, but the AI standardized to a heart rate of 75 beats per minute $(22.2$ vs. $26.8 \% ; P<0.0001)$ was lower on tonometric than oscillometric assessment, whereas the opposite was true for PWV (7.9 vs. $\left.7.4 \mathrm{~m} \mathrm{~s}^{-1} ; P=0.0002\right)$.

The differences between the tonometric and oscillometric estimates of the central hemodynamic variables (Figure 3 ) increased with the level of the measurement under the study for cSP $(r=0.37$; $P=0.0004), \quad$ AI $\quad(r=0.39 ; \quad P=0.0002) \quad$ and $\mathrm{PWV} \quad(r=0.39$; $P=0.0003)$, but not for $\mathrm{CDP}(r=0.15 ; P=0.17)$ and pulse pressure $(r=0.13 ; P=0.25)$. Supplementary Figure $\mathrm{S} 2$ shows the scatter plots of the oscillometric $v s$. the tonometric measurements. The correlation coefficients ranged from 0.45 to 0.77 . The regression lines all significantly deviated from the line of identity $(P<0.0001)$. The oscillometric measurements tended to be higher than the tonometric

Table 3 Central hemodynamic variables measured by tonometry or oscillometry at rest in 35 participants

\begin{tabular}{|c|c|c|c|c|c|c|c|}
\hline \multirow[b]{2}{*}{ Variable } & \multicolumn{2}{|c|}{ Meants.d. } & \multicolumn{2}{|c|}{ Absolute difference } & \multicolumn{2}{|c|}{ Relative difference } & \multirow[b]{2}{*}{$\mathrm{P}$} \\
\hline & $T M$ & $O M$ & Mean \pm s.d. & $95 \% \mathrm{Cl}$ & Mean \pm s.d. & $95 \% \mathrm{Cl}$ & \\
\hline Systolic BP, mm Hg & $105.6 \pm 17.1$ & $106.9 \pm 16.6$ & $-1.2 \pm 3.1$ & -2.3 to 0.2 & $-1.2 \pm 3.0$ & -2.3 to 0.2 & 0.22 \\
\hline Diastolic BP, mm Hg & $74.6 \pm 10.8$ & $74.7 \pm 11.3$ & $-0.1 \pm 2.4$ & -1.0 to 0.7 & $-0.1 \pm 3.0$ & -1.1 to 0.9 & 0.72 \\
\hline Pulse pressure, $\mathrm{mm} \mathrm{Hg}$ & $31.0 \pm 9.0$ & $32.1 \pm 7.4$ & $-1.1 \pm 4.1$ & -2.5 to 0.3 & $-4.7 \pm 13.2$ & -9.2 to -0.2 & 0.12 \\
\hline Al, \% & $21.1 \pm 13.0$ & $20.6 \pm 11.1$ & $0.5 \pm 9.5$ & -2.8 to 3.8 & $-7.6 \pm 58.0$ & -27.6 to 12.3 & 0.65 \\
\hline Al at $75 \mathrm{bpm}, \%$ & $15.1 \pm 13.4$ & $14.0 \pm 12.5$ & $1.1 \pm 11.5$ & -2.8 to 5.0 & $71.8 \pm 166.4$ & -36.7 to 220.3 & 0.57 \\
\hline $\mathrm{PWV}, \mathrm{ms}^{-1}$ & $7.3 \pm 1.9$ & $7.0 \pm 2.2$ & $0.3 \pm 1.1$ & -0.1 to 0.7 & $5.4 \pm 15.1$ & 0.1 to 10.7 & 0.11 \\
\hline
\end{tabular}

Abbreviations: Al, augmentation index; $\mathrm{BP}$, blood pressure; $\mathrm{Cl}$, confidence interval.

TM (average of two readings) and OM (average of four readings) refer to measurements obtained by the tonometric SphygmoCor technique at the radial artery or by the oscillometric Mobil-O-Graph monitor at the brachial artery, respectively. PWV is the carotid-femoral pulse wave velocity measured by TM (average of two readings) or aortic pulse wave velocity estimated by OM (average of four readings).

Relative difference was computed from paired readings as $(\mathrm{TM}-\mathrm{OM}) /[(\mathrm{TM}+\mathrm{OM}) / 2] \times 100$.

$P$-values are for the differences between TM and OM.

Table 4 Central hemodynamic variables measured by tonometry at rest and by oscillometry during daytime ambulatory monitoring in 83 participants

\begin{tabular}{|c|c|c|c|c|c|c|c|}
\hline \multirow[b]{2}{*}{ Variable } & \multicolumn{2}{|c|}{ Mean \pm s.d. } & \multicolumn{2}{|c|}{ Absolute difference } & \multicolumn{2}{|c|}{ Relative difference } & \multirow[b]{2}{*}{$P$} \\
\hline & $T M$ & $O M$ & Mean \pm s.d. & $95 \% \mathrm{Cl}$ & Mean \pm s.d. & $95 \% \mathrm{Cl}$ & \\
\hline Systolic BP, mm Hg & $115.4 \pm 14.5$ & $113.9 \pm 10.9$ & $1.4 \pm 10.0$ & -0.7 to 3.7 & $0.9 \pm 8.9$ & -1.0 to 2.9 & 0.19 \\
\hline Diastolic BP, mm Hg & $77.8 \pm 10.2$ & $81.9 \pm 9.2$ & $-4.0 \pm 7.3$ & -5.6 to -2.4 & $-5.3 \pm 9.2$ & -7.3 to -3.2 & $<0.0001$ \\
\hline Pulse pressure, $\mathrm{mm} \mathrm{Hg}$ & $37.6 \pm 7.4$ & $32.1 \pm 6.6$ & $5.5 \pm 7.4$ & 3.9 to 7.1 & $15.6 \pm 21.3$ & 10.9 to 20.0 & $<0.0001$ \\
\hline Al, \% & $26.5 \pm 12.6$ & $25.3 \pm 7.2$ & $0.7 \pm 9.3$ & -1.5 to 2.8 & $-13.2 \pm 65.5$ & -28.2 to 1.7 & 0.54 \\
\hline Al at $75 \mathrm{bpm}, \%$ & $22.2 \pm 11.5$ & $26.8 \pm 7.5$ & $-4.6 \pm 9.4$ & -6.6 to -2.5 & $-14.0 \pm 142.9$ & -45.6 to 17.6 & $<0.0001$ \\
\hline $\mathrm{PWV}, \mathrm{ms}^{-1}$ & $7.9 \pm 2.1$ & $7.4 \pm 1.6$ & $0.6 \pm 1.3$ & 0.3 to 0.9 & $6.5 \pm 15.1$ & 3.2 to 10.0 & 0.0002 \\
\hline
\end{tabular}

Abbreviations: Al, augmentation index; $\mathrm{BP}$, blood pressure; $\mathrm{Cl}$, confidence interval.

TM (average of two readings) and OM (average of daytime ambulatory readings) refer to measurements obtained by the tonometric SphygmoCor technique at the radial artery or by the oscillometric Mobil-O-Graph monitor at the brachial artery, respectively. PWV is the carotid-femoral pulse wave velocity measured by TM (average of two readings) or aortic pulse wave velocity estimated by OM (average of daytime ambulatory readings).

Relative difference was computed from paired readings as $(\mathrm{TM}-\mathrm{OM}) /[(\mathrm{TM}+\mathrm{OM}) / 2] \times 100$

$P$-values are for the differences between TM and OM. 

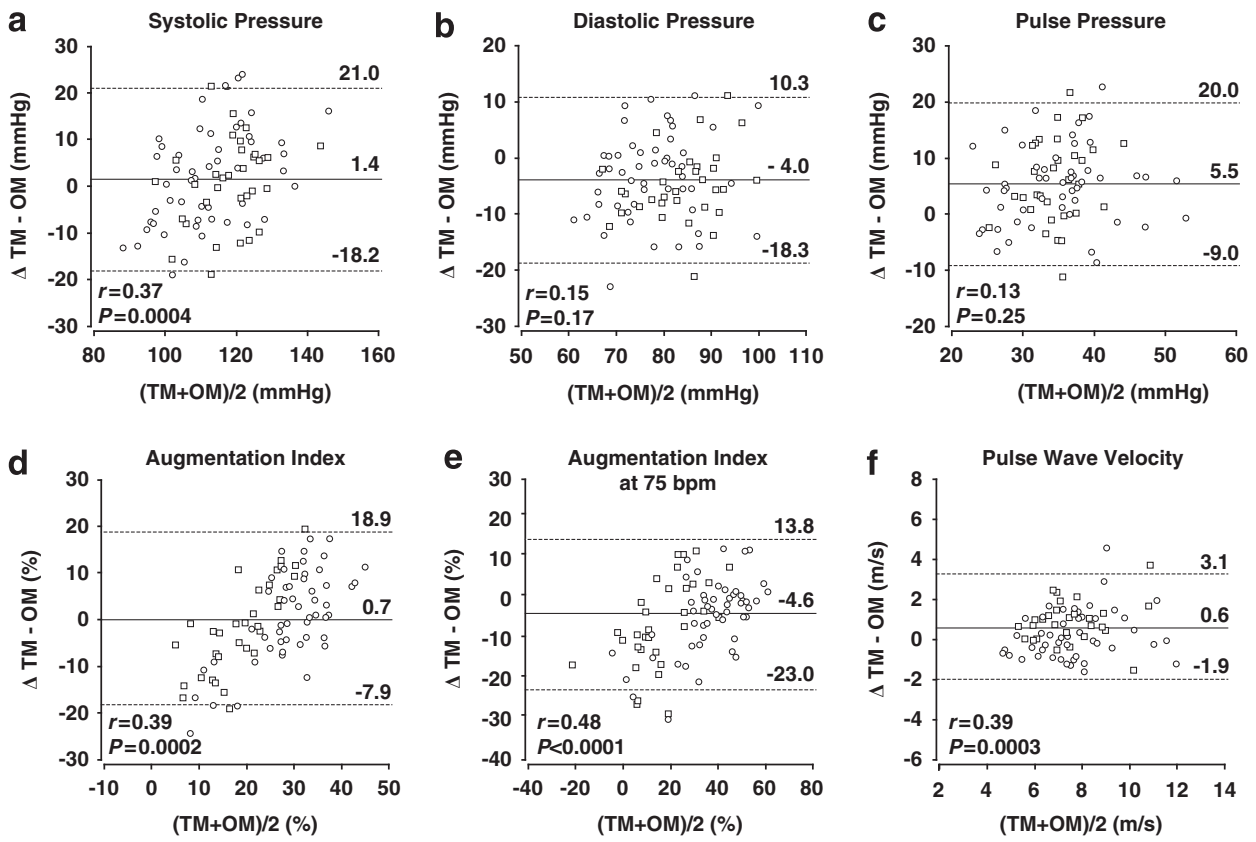

Figure 3 The Bland and Altman plots for the differences $(\Delta)$ of tonometric minus oscillometric estimates of (a) central systolic, (b) diastolic and (c) pulse pressures, the (d) crude and (e) standardised augmentation indices and (f) aortic pulse wave velocity. Plots include 51 women (circles) and 32 men (squares) The horizontal axis is the mean of the tonometric (TM) and oscillometric (OM) estimates within the individual subjects. TM (average of two readings) and $\mathrm{OM}$ (average of daytime ambulatory readings) refer to the measurements obtained by the tonometric SphygmoCor technique at the radial artery or by the oscillometric Mobil-O-Graph monitor at the brachial artery, respectively. The SphygmoCor measurements were calibrated by the brachial blood pressure obtained by means of the OMRON 705IT. $r$ and $P$-values indicate the correlation coefficient and its significance, respectively.
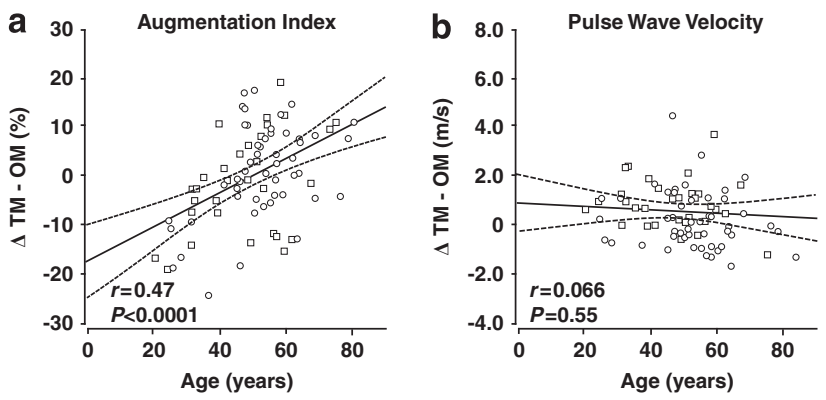

Figure 4 Scatter plots of tonometric minus oscillometric ( $y$ axis) vs. age $(x$ axis) estimates of (a) augmentation index and (b) pulse wave velocity. Plots include 51 women (circles) and 32 men (squares). The regression line with 95\% confidence interval and the line of identity are shown. $r$ indicates the correlation coefficient $( \pm \mathrm{SE})$. $P$-values are for the difference between $\beta$ and the line of identity $(\beta=1)$.

estimates at low values of the SphygmoCor measurements with the opposite trend at high values.

Figure 4 shows that the oscillometric estimates of the AI tended to be higher at younger ages, with the opposite at older ages $(r=0.47$; $P<0.0001)$. Tonometric minus oscillometric estimates for PWV were not correlated with age $(r=0.066 ; P=0.55)$.

To minimize the influence of time of day, we compared tonometric estimates with the first two and last two oscillometric measurements obtained at the initiation and completion of the 24-h ambulatory recordings. The Bland and Altman analysis appears in Supplementary Table S1 online. The results confirmed those reported in Table 4.

\section{DISCUSSION}

Arterial stiffness is an established risk factor predictive of cardiovascular complications over and beyond traditional risk factors. Systolic augmentation and pulse pressure in the central arteries and aortic PWV reflect stiffness of the arterial tree. A common characteristic of these indices is that they can only be measured with the subjects resting in the supine position and that they require observer training. The ARCSolver application, ${ }^{8}$ embedded in the validated Mobil-OGraph 24h PWA Monitor blood pressure monitor, ${ }^{6,7}$ represents an attempt to record indices of arterial stiffness under ambulatory conditions without the necessity of training observers. In the current study, we compared the central hemodynamic measurements obtained by the Mobil-O-Graph at rest and under ambulatory conditions with those obtained by the SphygmoCor at rest. The key finding of our study was that, irrespective of measurement conditions, brachial oscillometry by the Mobil-O-Graph, compared with the established radial tonometric method, provided comparable estimates of cSP and systolic augmentation uncorrected for heart rate. Central PWV was slightly lower on oscillometric than tonometric assessment.

The arterial pressure waveform is a composite of the forward pressure wave created by the ventricular contraction and a reflected wave. ${ }^{15}$ In stiff arteries, PWV rises and reflected waves return earlier to the central arteries and augment the systolic pressure of the forward wave. ${ }^{15}$ The AI quantifies this phenomenon. The aortic pressure waveform can be estimated from the radial waveform by means of a transfer function or it can be approximated from the common carotid waveform. We chose to use radial tonometry, because the radial artery is well supported by bony tissue, making optimal applanation easier to achieve. Moreover, the transfer function implemented in the SphygmoCor software has been extensively validated in invasive studies. $^{3-5}$ The age-related changes in peripheral and central blood pressures in large population studies, ${ }^{16,17}$ as recorded by the SphygmoCor, ran a course as predicted by the physiological principles underlying systolic 
augmentation ${ }^{15}$ and constitute a large body of evidence validating this approach over and beyond the available invasive studies. ${ }^{3-5}$ Reference values for the central hemodynamic variables as obtained by the SphygmoCor are available for Asians, ${ }^{18}$ Blacks of native African origin, ${ }^{19}$ and Europeans. ${ }^{20}$ The SphygmoCor approach has been widely applied in randomized clinical trials with intermediate and hard outcomes. ${ }^{21}$ These arguments support the use of tonometry by the SphygmoCor approach as a reference method in the present study.

The ARCSolver algorithm, as implemented in Mobil-O-Graph 24h PWA Monitor, reconstructs the central pulse wave by applying a transfer function ${ }^{8}$ and computes the central systolic AI and aortic PWV. Although the Mobil-O-Graph 24h Monitor has been properly validated for blood pressure measurement according to commonly accepted and standardised protocols, ${ }^{6,7}$ only two small studies, one based on impedance cardiography, ${ }^{12}$ and another on thermodilution, ${ }^{12}$ provided a preliminary validation of the estimates of cardiac output, which is only a secondary variable generated by the ARCSolver software.

Weber et al. ${ }^{22}$ compared cSP, calculated with a transfer-function like method (ARCSolver algorithm), using waveforms recorded with a regular oscillometric cuff suitable for ambulatory measurements, with simultaneous high-fidelity invasive recordings, and with noninvasive estimations using a validated device (SphygmoCor), operating with radial tonometry and a generalized transfer function. For the invasive study, Weber recruited 30 patients undergoing elective coronary angiography for suspected coronary artery disease. For the noninvasive comparison, he recruited 111 inpatients as well as healthy volunteers. Exclusion criteria for both studies were unstable clinical conditions, arrhythmias that would disturb the regular rhythm required during the pulse recordings and significant valvular heart disease. Medicated patients were kept on drug treatment. Both studies revealed good agreement between the oscillometric cuff-based cSP and the comparator. In the invasive study, mean difference between oscillometric cuff/ARCSolver-based and invasive cSPs was $3.0 \mathrm{~mm} \mathrm{Hg}$ (s.d. $6.0 \mathrm{~mm} \mathrm{Hg}$ ) with invasive calibration of the brachial waveforms and $-3.0 \mathrm{~mm} \mathrm{Hg}$ (s.d.: $9.5 \mathrm{~mm} \mathrm{Hg}$ ) with noninvasive calibration. Results were similar when the reference method (radial tonometry/transfer function) was compared with invasive measurements. In the noninvasive study, composed of 111 patients, the mean difference between oscillometric cuff/ARCSolver-derived and radial tonometry/transfer functionderived cSPs was $-0.5 \mathrm{~mm} \mathrm{Hg}$ (s.d. $4.7 \mathrm{~mm} \mathrm{Hg}$ ).

Under all measurement conditions, brachial oscillometry compared with the established radial tonometric method provided similar estimates of cSP. In the studies at rest under controlled laboratory conditions, cDP and pulse pressure were similar on both measurement techniques, whereas under ambulatory conditions cDP was higher and pulse pressure was lower on oscillometric compared with tonometric assessment. Several factors might explain the differences. First, the SphygmoCor device was calibrated by the brachial blood pressure readings obtained by different devices: the Mobil-O-Graph in the studies exclusively at rest and the OMRON 705IT in the studies at rest and under ambulatory conditions. Second, physical activity and psycho-emotional stress influenced the ambulatory values. Third, the blood pressure responses that follow postural change from the supine to the standing position are since long established. ${ }^{23}$ Upon standing, blood begins to pool in the venous system. This causes a decrease in venous return and a drop in cardiac output. Systemic blood pressure falls with cardiac output. The baroreceptor reflex then activates the sympathetic nervous system, promotes vasoconstriction and increases heart rate, which stabilizes cardiac output. Mean blood pressure is maintained by an increase in total peripheral resistance. Overall, the response to standing is usually a very slight reduction $(<4 \mathrm{~mm} \mathrm{Hg})$ in SBP and slight increase $(<10 \mathrm{~mm} \mathrm{Hg})$ in DBP. ${ }^{24,25}$

In the $\mathrm{R}+\mathrm{A}$ study, $\mathrm{PWV}$ was higher on tonometric than oscillometric assessment, with a similar trend in the R study. Several factors can explain the differences averaging $0.6 \mathrm{~m} \mathrm{~s}^{-1}$ in the $\mathrm{R}+\mathrm{A}$ study and $0.5 \mathrm{~ms}^{-1}$ at the start and completion of the 24-h ambulatory monitoring. The tonometric assessment by the SphygmoCor requires measurement of travel distance and provides carotid-femoral PWV. The oscillometric estimates are for aortic PWV and do not necessitate measurement of travel distance. Carotidfemoral PWV cannot be equated to aortic PWV. ${ }^{26,27}$ Weber et al. ${ }^{27}$ measured aortic PWV from the ascending aorta to the bifurcation invasively in 378 patients undergoing coronary angiography as well as carotid-femoral PWV non-invasively, using the SphygmoCor system. Body height and the non-invasively measured travel distance decreased with higher age. By contrast, the invasively measured travel distance (catheter length), tended to increase with age, probably owing to an increase in aortic length or kinking. ${ }^{27}$ As a result, the difference of carotid-femoral minus aortic travel distance decreased with age. Carotid-femoral as well as aortic travel time shortened with higher age, but the shortening was more pronounced for aortic travel time. These trends resulted in net differences of carotid-femoral minus aortic PWV of $0.7 \mathrm{~m} \mathrm{~s}^{-1}$ in the youngest patients ( $<45$ years), decreasing up to $-1 \mathrm{~m} \mathrm{~s}^{-1}$ in the oldest ( $>75$ years) patients. ${ }^{27}$ Weber et al. ${ }^{26}$ also demonstrated that among 135 patients (mean age, 60.3 years) the method of subtracting carotid-suprasternal notch distance from suprasternal notch-femoral distance as applied in the current study provided the best agreement with invasive assessments of the travel path based on catheter length. The average difference was only $0.9 \mathrm{~cm}$.

Pulse wave analysis derives the central from the peripheral blood pressure waves and is calibrated based on brachial blood pressure. As observed in the current study, it therefore follows that the central blood pressure must follow a diurnal course similar to that of the peripheral brachial blood pressure. Heart rate decreases at night. Because the central AI is inversely correlated with heart rate, it increases at night. Standardization of the AI to a heart rate of 75 beats per minute removed the diurnal profile. PWV depends on cardiac output and blood pressure. Because both decrease at night, central PWV was slightly but significantly lower at night than during the day.

The present study must be interpreted within the context of its limitations and strengths. First, the current study does not allow to ascertain whether the tonometric or the oscillometric technique provides estimates of the central hemodynamic variables that are closest to the true invasively measured values. However, invasive studies are not readily ethically acceptable in volunteers and are almost impossible to implement under ambulatory conditions. Second, we recruited volunteers among the employees and patients of the Hospital de Clínicas, Montevideo. These volunteers, many of them on antihypertensive treatment, were self-selected and are not representative for a general population. Third, pulse wave analysis was used to CPP and central AI. Such an approach may have led to a small degree of error in central pressure estimation, but the transfer function involved has been previously invasively validated for the SphygmoCor device and the Mobil-O-Graph. The studies at rest have several strong points. All the measurements were obtained in a controlled laboratory environment, with participants resting in the supine position. The SphygmoCor was calibrated using the brachial blood pressure as measured by the Mobil-O-Graph. The intraobserver intra-session and inter-session reproducibility was high.

In conclusion, our study suggests that the ambulatory non-invasive assessment of central hemodynamic variables is feasible. The 
ARCSolver algorithm underwent preliminary validation in terms of a composite cardiovascular outcome in patients with suspected coronary heart disease undergoing coronarography. ${ }^{28}$ However, further observations in diverse populations are required before ambulatory assessment of the central hemodynamic variables can make it to clinical practice. In particular, clinicians need to know the distribution of these measurements in women and men across different age groups. Prospective studies must generate the outcome data that the diurnal profile of the central hemodynamic measurements provided by the Mobil-O-Graph 24h PWA Monitor adds to risk stratification. In the mean time, population studies would also allow investigation of the cross-sectional associations of intermediate signs of target organ damage with the circadian variation in the central hemodynamic measurements over and beyond the 24-h brachial blood pressure.

\section{CONFLICT OF INTEREST}

The authors declare no conflict of interest.

\section{ACKNOWLEDGEMENTS}

Atcor Medical (West Ryde, New South Wales, Australia) and IEM GmbH (Stolberg, Germany) provided the equipment free of charge for use in the Genotipo, Fenotipo y Ambiente de la Hipertensión Arterial en Uruguay (GEFA-HT-UY) study. The European Union (grants IC15-CT98-0329EPOGH, HEALTH-F4-2007-201550 HyperGenes, and HEALTH-2011.2.4.2-2 EU-MASCARA) and the Fonds voor Wetenschappelijk Onderzoek Vlaanderen, Brussels, Belgium (grants G.0575.06 and G.0734.09) supported the Studies Coordinating Center (Leuven, Belgium). The Programa para la Formación y Fortalecimiento de los Recursos Humanos de los Prestadores Públicos de Servicios de Salud, Unidades Docentes Asistenciales gave support to the Unidad de Hipertensión Arterial.

1 Hansen TW, Staessen JA, Torp-Pedersen C, Rasmussen S, Thijs L, Ibsen H, Jeppesen J. Prognostic value of aortic pulse wave velocity as index of arterial stiffness in the general population. Circulation 2006; 113: 664-670.

2 Laurent S, Cockcroft J, Bortel LV, Boutouyrie P, Giannattasio C, Hayoz D, Pannier B, Vlachopoulos C, Wilkinson I, Struijker-Boudier H; European Network for Noninvasive Investigation of Large Arteries. Expert consensus document on arterial stiffness: methodological issues and clinical applications. Eur Heart J 2006; 27: 2588-2605

3 Pauca AL, O'Rourke M, Kon ND. Prospective evaluation of a method for estimating ascending aortic pressure from the radial artery pressure waveform. Hypertension 2001; 38: 932-937.

4 Chen CH, Ting CT, Nussbacher A, Nevo E, Kass DA, Pak P, Wang SP, Chang MS, Yin FC. Validation of carotid artery tonometry as a means of estimating augmentation index of ascending aortic pressure. Hypertension 1996; 27: 168-175.

5 Chen CH, Nevo E, Fetics B, Pak PH, Yin FC, Maughan WL, Kass DA. Estimation of central aortic pressure waveform by mathematical transformation of radial tonometry pressure. Validation of generalized transfer function. Circulation 1997; 95: 1827-1836.

6 Weiß W, Tölle M, Zidek W, van der Giet M. Validation of the mobil-O-Graph: 24h-blood pressure measurment device. Blood Press Monit 2010; 15: 225-228.

7 Franssen PML, Imholz BPM. Evaluation of the Mobil-0-Graph new generation ABPM device using the ESH criteria. Blood Press Monit 2010; 15: 229-231.
8 Wassertheurer S, Kropf J, Weber T, van der Giet M, Baulmann J, Ammer M, Hametner B, Mayer CC, Eber B, Magometschnigg D. A new oscillometric method for pulse wave analysis: comparison with a common tonometric method. J Hum Hypertens 2010; 24: 498-504.

9 Staessen JA, Bieniaszewski L, O'Brien ET, Imai Y, Fagard R. An epidemiological approach to ambulatory blood pressure monitoring: the Belgian population study. Blood Press Monit 1996; 1: 13-26.

10 Thijs L, Staessen J, Fagard R. Analysis of the diurnal blood pressure curve. High Blood Press Cardiovasc Prev 1992; 1: 17-28.

11 Chirinos JA, Segers P. Noninvasive evaluation of left ventricular afterload: Part 2: Arterial presssure-flow and pressure-volume relations in humans. Hypertension 2010; 56: 563-570.

12 Wassertheurer S, Mayer C, Breitenecker F. Modeling arteral and left ventricular coupling for non-invasive measurements. Simul Model Pract Theory 2008; 16: 988-997.

13 Wilkinson IB, McEniery CM, Schillaci G, Boutouyrie P, Segers P, Donald A, Chowienczyk PJ, On behalf of the ARTERY Society. ARTERY Society guidelines for validation of non-invasive haemodynamic measurement devices: Part 1, arterial pulse wave velocity. Artery Res 2010; 4: 34-40.

14 Bland JM, Altman DG. Statistical methods for assessing agreement between two methods of clinical measurement. Lancet 1986; 1: 307-310.

15 O'Rourke MF, Hashimoto J. Mechanical factors in arterial aging. A clinical perspective. J Am Coll Cardiol 2007; 50: 1-13.

16 McEniery CM, Yasmin, McDonnell B, Munnery M, Wallace SM, Rowe CV, Cockcroft JR, Wilkinson IB; Anglo-Cardiff Collaborative Trial Investigators. Central pressure: variability and impact of cardiovascular risk factors: the Anglo-Cardiff Collaborative Trial II. Hypertension 2008; 51: 1476-1482.

17 Li Y, Staessen JA, Sheng CS, Huang QF, O'Rourke M, Wang JG. Age dependency of peripheral and central systolic pressres: cross-sectional and longitudinal observations in a Chinese population. Hypertens Res 2012; 35: 115-122.

$18 \mathrm{Li}$ Y, Staessen JA, Li LH, Huang QF, Lu L, Wang JG. Reference values for the arterial pulse wave in Chinese. Am J Hypertens 2008; 21: 668-673.

19 Shiburi CP, Staessen JA, Maseko M, Wojciechowska W, Thijs L, Van Bortel LM, Woodiwiss AJ, Norton GR. Reference values for SphygmoCor measurements in South Africans of African ancestry. Am J Hypertens 2006; 19: 40-46.

20 Wojciechowska W, Staessen JA, Nawrot T, Cwynar M, Seidlerová J, Stolarz K, Gasowski J, Tichá M, Richart T, Thijs L, Grodzicki T, Kawecka-Jaszcz K, Filipovský J; European Project on Genes in Hypertension (EPOGH) Investigators. Reference values in White Europeans for the arterial pulse wave recorded by means of the ShygmoCor device. Hypertens Res 2006; 29: 475-483.

21 Williams B, Lacy PS, Thom SM, Cruickshank K, Stanton A, Collier D, Hughes AD, Thurston H, O'Rourke M. Differential impact of blood pressure-lowering drugs on central aortic pressure and clinical outcomes: principal results of the Conduit Artery Function Evaluation ( CAFE ) study. Circulation 2006; 113: 1213-1225.

22 Weber T, Wassertheurer S, Rammer M, Maurer E, Hametner B, Mayer CC, Kropf J, Eber B. Validation of a brachial cuff-based method for estimating central systolic blood pressure. Hypertension 2011; 58: 825-832.

23 MacWilliam JA. Postural effects on heart-rate and blood-pressure. Quat J Exp Physiol 1933; XXIII: 1-33.

24 Borst C, van Brederode JFM, Wieling W, van Montfrans GA. Mechanisms of initial blood pressure response to postural change. Clin Sci 1984; 67: 321-327.

25 Nardo CJ, Chambless LE, Light KC, Rosamond WD, Sharrett AR, Tell GS, Heiss G. Descriptive epidemiologie of blood pressure response to change in body position. The ARIC Study. Hypertension 1999; 33: 1123-1129.

26 Weber T, Ammer M, Rammer M, Adji A, O'Rourke MF, Wassertheurer S, Rosenkranz S, Eber B. Noninvasive determination of carotid-femoral pulse wave velocity depends critically on assessment of travel distance: a comparison with invasive measurement. J Hypertens 2009; 27: 1624-1630.

27 Weber T, Ammer M, Rammer M, O'Rourke M, Eber B. Age-dependent differences between aortic and carotid-femoral pulse wave velocity. J.Hypertens 2010; 28(e-supplementum A): e418-e419.

28 Weber T, Ammer M, Biber C, Windpessl M, Wassertheurer S, Hametner B, Mayer C, Kropf J, Rammer M, Lassnig E, Eber B. Arterial wave reflection and arterial stiffness independently predict cardiovascular events. J.Hypertens 2010; 28 (e-supplementum A): e579.

Supplementary Information accompanies the paper on Hypertension Research website (http://www.nature.com/hr) 